Clinical Medicine: Case Report

Poster

Abstract ID: 69

\title{
Recontruction of coronoid process using autologous tricortical iliac bone graft after persistent traumatic elbow dislocation- case report
}

Khairul Nizam Siron | Ardilla Hanim Abdul Razak | Mohd Shukrimi Awang | Aminuddin Che Ahmad

Department of Orthopaedics, Traumatology \& Rehabilitation, Kulliyyah of Medicine, International Islamic University Malaysia

Coronoid fractures are challenging to manage and rarely isolated. It's always associated with three instability patterns of the elbow, which is the terrible triads, varus posteromedial rotatory instability and transolecranon fracture- dislocation. We have performed an open reduction and reconstruction of the coronoid process using autologous tricortical iliac bone graft for one patient after persistent elbow dislocation in malunited type III coronoid fracture. The main goal of management of coronoid fracture with elbow dislocation is to maintain the joint stability and acceptable elbow functions. Through understanding the pattern of injuries, proper investigation and approach could improve the outcome of surgery. We believe that reconstructing coronoid process using tricortical iliac bone graft provides acceptable stable elbow joint and good functional flexion arc of motion.

KEYWORDS: Coronoid process, tricortical iliac bone graft 